\title{
La búsqueda de la realidad o de la verdad: una aproximación a partir de la teoría sociológica
}

\author{
THE SEARCH FOR TRUTH OR REALITY: AN APPROACH FROM SOCIOLOGICAL THEORY
}

Dr. Josep Vidal (josevidal@ufpa.br) Núcleo Altos Estudios Amazónicos, Universidade Federal do Pará (Belém, Brasil)

\begin{abstract}
The aim of this article is to think the relationship between the search for reality or the truth with methodology, by asking some questions to different theoretical perspectives and by analyzing different answers given by the authors and theories. From the sociological theoretical perspective, it deals with two levels of thought with methodological implications. The first refers to the methodological implications arising from the search for reality or truth. The second, epistemological, refers to the theoretical and paradigmatic duality between subjective capacity decisions against the constriction of the subjects by the structures.
\end{abstract}

Keywords: methodology, social science, reality, truth, social theory.

\section{Resumen}

El objetivo de este artículo es pensar el nexo de la búsqueda de la realidad o de la verdad con la metodología, al plantear preguntas a diversas corrientes teóricas y analizar las respuestas de cada autor o corriente al respecto. Desde la perspectiva teórica sociológica, se abordan dos niveles de reflexión con implicaciones metodológicas. El primero, hace referencia a las implicaciones metodológicas derivadas de la búsqueda de la realidad o de la verdad. El segundo, de carácter epistemológico, hace referencia a la dualidad teórica y paradigmática existente entre la capacidad subjetiva de decisión frente a la constricción de los sujetos por parte de las estructuras.

Palabras clave: metodología, ciencias sociales, realidad, verdad, teoría social.

\section{Introducción}

Cuestiones de carácter metafísico y ontológico, como son la búsqueda de la realidad o de la verdad, han sido poco debatidas con la necesaria profundidad y claridad en las corrientes sociológicas contemporáneas y en los manuales de metodología existentes. A pesar de ello, se trata de un problema central de carácter ontológico de la cual derivan otras cuestiones epistemológicas con implicaciones para la metodología en la búsqueda del conocimiento. Diferente es su debate académico en la filosofía y en las ciencias sociales y humanas alemanas, en la que el concepto de verdad y sus implicaciones metafísicas es un tema recurrente y de constante debate (Falk, Franze, entre otros), así como también sus implicaciones con lo que se denomina realidad (Knaller y Müller, Janew, entre otros).

Debatir estas ideas, y sus implicaciones metodológicas, no constituye un debate exclusivamente metafísico, sino que a nuestro entender, constituye la parte decisiva de la lógica que rige el enfoque teórico y la metodología de la observación. El artículo tiene como objetivo debatir cómo las diversas corrientes de la sociología contemporánea 
abordan estos conceptos y cuáles son las implicaciones metodológicas, derivadas de cuestiones epistemológicas y ontológicas que rigen la estrategia y finalidad de la adquisición de conocimiento en las ciencias sociales. A partir de estas premisas, dos hipótesis teóricas de carácter ontológico y epistemológico configuran el artículo.

La primera hipótesis de carácter ontológico hace referencia directa a la interpretación de la realidad o de la verdad en la sociología en sus aspectos filosóficos, de la siguiente forma: la aparición de la corriente sociológica del interaccionismo simbólico a finales de la década de los años 60 , ha propiciado un cambio metodológico en las ciencias sociales y humanas sobre cómo se entiende la realidad y la verdad, de tal forma que la comprensión inicial de una realidad objetiva, se ha desplazado hacia una comprensión de una realidad interpretada y construida subjetivamente. Con ello se pone en cuestión la validez de los hechos objetivos. El cambio paradigmático se debe al asimilar el principio ontológico de los juicios sintéticos a priori propuestos por Kant, y que posteriormente fueron expuestos en lenguaje sociológico por Weber, y por las corrientes interpretativas. Este paso ha tenido implicaciones en la tradicional hegemonía empirista de los métodos cuantitativos. Aceptar la hipótesis tiene implicaciones epistemológicas. La tradicional concepción de que los sujetos están determinados por las estructuras, es puesta en duda por el principio interaccionista: los sujetos son producto y productores de las estructuras (Berger y Luckmann). Posteriores lecturas construccionistas han radicalizado la tesis, de tal forma que Peter Berger llega a afirmar: "solamente un bárbaro intelectual es capaz de afirmar que la realidad es únicamente lo que podemos ver mediante métodos científicos" (Berger 1985:40).

La segunda hipótesis abre el debate teórico entorno al cambio del concepto de autopoiesis propuesto por Francisco Valera y Humberto Maturana y más tarde adaptado a la sociología por Niklas Luhmann. Esta adaptación a la sociedad significa un paso epistemológico con implicaciones metodológicas. La argumentación es similar a la dada por la sociobiología y la perspectiva neurofisiológica de Francisco Valera y Humberto Maturana, por ejemplo, cuando Maturana sostiene que "todo lo dicho", o sea, resultado del proceso de la observación, "es dicho por un observador" (Maturana y Varela 1985:35). Se cuestiona metafísicamente la validez universal de las sentencias y observaciones, al depender estas de lo que hace el observador, hasta el punto de afirmar, "sin el observador no hay nada". El hecho de replantear el concepto de observador y observación, estableciendo una diferencia en la teoría de sistemas autorreferenciales o autopoiéticos de Niklas Luhmann, no es contemplado en los conceptos clásicos de la filosofía.

El ejercicio epistemológico propuesto requiere iniciar un contraste teórico entre diferentes cosmovisiones y axiomas entre las diversas corrientes de la sociología actual. Sin embargo, antes de iniciar la exposición teórica con representantes de diferentes paradigmas o cosmovisiones, conviene clarificar el método y las reglas con que se realizará, posibilitándose dos marcos de debate:

a) La exposición de los paradigmas de diferentes teorías, con el objetivo de mostrar los puntos fuertes y flaquezas de cada una de ellas, en cómo abordan empíricamente la procura de la realidad o de la verdad. Con ello se establecen conceptos previamente definidos para su posterior comparación.

b) Iniciar el debate a partir de la idea central de este artículo, o sea, el nexo de la búsqueda de la realidad o de la verdad con la metodología, al plantear preguntas a las diversas corrientes teóricas y analizar las respuestas de cada autor o corriente.

En este artículo nos hemos inclinado por la segunda opción, al permitir formular cuestiones sin una definición conceptual realizada previamente. 


\section{La visión del ser humano en la investigación en las ciencias sociales y humanas}

La visión del ser humano en la investigación varía en cada época histórica, dependiendo de cuestiones básicas que se plantean los científicos y los filósofos como: ¿qué es la realidad social?, ¿qué son los hechos sociales? Aunque estas preguntas presenten cierta similitud, las respuestas varían profundamente y en consecuencia tienen efectos en el método adoptado para la investigación. Dos perspectivas pueden anticiparse: una perspectiva y metodología positivista y la interpretativa. Perspectivas, como veremos, estrechamente vinculadas con el debate epistemológico entorno a la forma de entender los procesos y los fenómenos sociales entre el enfoque idiográfico y el nomotético.

Para intentar responder la pregunta central de este artículo, la búsqueda de la realidad o de la verdad en la sociología contemporánea, es necesario plantear una serie de cuestiones en dos niveles: un primer nivel epistemológico y un segundo ontológico. El debate epistemológico, o sea, los métodos que llevan a la obtención del conocimiento, en este caso el axioma referente a la acción y estructura, es considerado como la "cuestión básica de la teoría social moderna" (Archer 1988: ix). En las corrientes de la sociología y las ciencias humanas actuales, sobresale habitualmente la cuestión entorno al debate epistemológico: ¿el ser humano es libre en sus decisiones o está determinado por las estructuras? Aunque responder a esta cuestión pueda incidir más en el nivel metafísico que en el sociológico y metodológico, la respuesta tiene implicaciones para la investigación en las ciencias en general, y, en particular, en las sociales.

Los sociólogos han dado respuestas de síntesis, que se dirigen a una integración (Giddens, Bourdieu, Habermas, Burt), y con una perspectiva feminista (Lengermann y Niebrugge-Brantle), entre otros. A pesar de estos esfuerzos teóricos para superar el antagonismo teórico, la pregunta ontológica ¿qué es conocer? sigue siendo actual en la procura de la realidad, o de la verdad. Para resolver los problemas de carácter metafísico y epistemológico expuestos, sobresalen dos cuestiones centrales. Primera, referente a la forma de entender la investigación entre idiográfica, o sea, el estudio de los sucesos cambiantes e irrepetibles, o nomotética, la procura de las leyes lógicas y la procura causal e inmutable de los hechos, a partir de la observación de la naturaleza. La segunda, referente a la cosmovisión y al tipo de perspectiva que orienta la lógica de la investigación: positivista, interpretativa, dialéctica, sistémica y sistémica autorreferencial.

En el nivel ontológico, las cuestiones hacen referencia al entendimiento de la realidad a partir de entender el ser, en cuanto ser, y el entorno: ¿qué entendemos por lo que denominamos realidad?, ¿cómo nos relacionamos con la realidad? En el momento de interpretar los hechos y los fenómenos sociales, y de desarrollar una investigación o un estudio interdisciplinario, aparecen cuestiones importantes, a partir de diferentes presupuestos ontológicos, los cuales marcan la teoría que el investigador escoge y la investigación social. Las respuestas para la cuestión ontológica, ¿la realidad social es externa al individuo o es producto de la conciencia individual? han marcado el debate filosófico entre científicos sociales y filósofos, ya que la forma de concebir este presupuesto tiene implicaciones en la estrategia, método y paradigma de investigación y en el tipo de información e interpretación de los dados que realizará el investigador. La forma en que se interpreta y relaciona el ser humano en una observación, influye directamente en la manera de exponer los pasos metodológicos siguientes, los problemas y las cuestiones que deben ser respondidas, como ya advirtieron Durkheim, Weber y más tarde Luhmann, aunque desde perspectivas diferentes.

Las cuestiones formuladas forman parte de la visión e imagen de la tradición ontológica anclada en la sociedad occidental, puesto que estos pensamientos son vertientes de la ontología de la totalidad, característica del pensamiento europeo, que privilegia "el ser-mismo" en detrimento del discurso del Otro. La obra de Descartes ya destaca esta tendencia con el destaque del "Yo", más tarde en Kant con la "consciencia trascendental" y en Husserl con la "conciencia intencional", ideas que se reflejarán posteriormente en toda la sociología occidental. Tampoco el 
debate entre el pensamiento holístico frente al asimétrico es nuevo en el pensamiento sociológico. Ya Frederich Engels, en Anti-Dühring o la revolución de la ciencia, al plantear cuestiones sociales y políticas se preguntaba: ¿El mundo objetivo crea el pensamiento o es el pensamiento que crea la materia? La respuesta corresponde a la existencia de un mundo externo explicado a través del empirismo. Por otro lado, el pensamiento holístico parte de la comprensión global de los hechos para derivar hacia aspectos de las realidades que forman estos procesos expuestos en los "principios del pensamiento holístico" de Morín (se expone en apartado 2.4).

\section{2. ¿La búsqueda de la realidad o de la verdad?}

Las preguntas ¿qué es conocer? y ¿qué es la verdad? han preocupado a los filósofos desde a Grecia clásica hasta los días de hoy. No es en vano, puesto que los científicos que se basan en corrientes fenomenológicas, hermenéuticas y autorreferenciales (en el sentido dado por la sociobiología), dudan de la existencia de una realidad universal y procuran una forma interpretativa o subjetiva de la verdad.

El debate epistemológico y las controversias no surgen con el inicio de la sociología, sino que hay que buscarlas entre los pensadores de la Grecia clásica. Aristóteles se fundamenta en dos premisas. La primera es que la verdad estaría en el pensamiento o en el lenguaje y no en otra cosa. La segunda premisa se basa en el hecho de que la verdad, o su reificación, es externa a ella. Refiriéndose a estos primeros pensadores, Ferrater Mora en el Diccionario de Filosofía escribe que, para ellos, la verdad es igual a la realidad y esta es igual al pensamiento, aunque este pensamiento debe tener una visión inteligible, o sea, un método riguroso.

Una forma ilustrativa de exponer qué entendemos por verdad, es referirnos a la alegoría de la caverna de Platón. Platón imagina que los prisioneros que están agrupados en un muro en el interior de la caverna, están allá desde su nacimiento y su cabeza está dirigida solo para el interior de la caverna. Atrás de ellos se encuentra un fuego y un camino para salir. A través del camino circulan personas y animales que proyectan sus sombras en la pared del fondo de la caverna. Los prisioneros dentro de la caverna apenas pueden percibir sus sombras, de tal forma que acreditan que estas sombras son reales, siendo las únicas que han visto durante toda su vida. Un día, uno de los prisioneros es liberado, pudiendo salir de la caverna. Las llamas y el sol lo deslumbraran completamente, poco a poco se acostumbra a ver el mundo real alrededor de él. Este hecho abre un proceso de diferenciar los objetos y de reconocer la pobreza en la cual había sido sometido durante muchos años. Posteriormente, el prisionero es forzado a volver a su posición original en la caverna, viendo y distinguiendo el mundo de las sombras del mundo real que había visto. Sus compañeros, entretanto, siguen creyendo que el mundo real es el mundo de sombras, pues nunca tuvieron la oportunidad de experimentar el mundo real. La parábola de Platón muestra que la mayoría de la humanidad se contenta en vivir en un mundo de apariencia. Pero también podemos extraer otra lección: la verdad y la luz son la única realidad, porque ella es todo lo que vemos. En el idealismo, la consciencia puede equivaler a la luz de la caverna.

Con el mito de la caverna de Platón es posible percibir diferentes interpretaciones y la importancia que adquiere la convivencia. La mayoría de los filósofos concuerda que con la idea de verdad, se trata de una interpretación mental de la realidad transmitida por los sentidos. De ser así, la interpretación subjetiva de la realidad filtrada a través de los sentidos nos muestra la verdad, en su caso inclusive posibles verdades. Entretanto, debemos recordar que la interpretación mental incluye creencias, valores y, en última instancia, la consciencia, porque pueden engañarnos una vez que filtran la verdad a partir de nuestra subjetividad, construyendo una verdad solamente para nosotros mismos. Definiciones normativas nos ofrecen una aproximación a la idea que tratamos. El Dicionário Aurélio define la verdad como "la identidad de una representación con la realidad representada", y amplía que se trata de un "cierto principio constante". Similar es la definición dada por el Deutsche Duden der Sprache en el que la verdad es descrita como: "la realidad de las situaciones dadas; la auténtica situación". En ambas definiciones, la verdad aparece como 
reflejo de la realidad de las situaciones. No nos proporciona una clarificación sobre su búsqueda, pues, en este caso, se deberá profundizar las raíces fenomenológicas del concepto.

¿En qué premisas y significados se basa la filosofía? Entre las corrientes de pensamiento filosófico y su vinculación con las ciencias sociales, destacamos la filosofía analítica (el análisis del significado de los enunciados) y la filosofía basada en el racionalismo crítico (en oposición directa al positivismo lógico).

La filosofía analítica, cuyas raíces se encuentran en el positivismo denominado operacionista y en el Círculo de Viena, establece una síntesis de pensamiento entre la ética y la lógica. Se debe al filósofo Ludwig Wittgenstein, en el Tractatus Lógico-Philosophicus, el planteamiento de la vinculación estructural entre el lenguaje y el mundo. Considera los hechos como "estados de cosas" y extrae la idea central de la teoría de la figuración y de la verdad. Una proposición será significativa o tiene sentido en la medida que representa un estado de cosas lógicamente posible. Puede ocurrir que un significado sea fragmentado en varios sentidos, o el mismo desintegrarse en la incoherencia. Profundiza el concepto de verdad y la relación con las proposiciones llegando a preguntarse: "¿Qué es y qué significa: la verdad de una proposición sea cierta?" La verdad es relativizada a partir de la interpretación subjetiva de los hechos. La utilización que realiza Wittgenstein de la expresión "verdadero o falso" es un tanto "engañadora" y equivale a decir "ajustarse al hecho o no" y lo que verdaderamente está en cuestión es el significado de "ajustarse". Uno de los requisitos de la búsqueda de la verdad constituye la identidad. La identidad de un significado tiene lugar a partir de diferentes interpretaciones que las personas atribuyen al significado: "Ios límites de un lenguaje son los límites de mi mundo". La identidad del significado vuelve posible la verdad.

Además de la filosofía analítica de Wittgenstein, se desarrollaron diversas corrientes. Una filosofía denominada continental representada por Martin Heidegger y la posibilidad de trascendencia histórica y cultural en busca de una situación social de Hans-Georg Gadamer, así como la caracterización del método científico, el dominio de la falsabilidad, y el criterio de comprobación racionalista de Karl Popper.

\subsection{El falsacionismo}

La perspectiva racionalista y empirista de Karl Popper se basa en hechos materiales. Con una perspectiva crítica frente al inductivismo y con base en un racionalismo crítico, Popper entiende como verdad "la correspondencia con los hechos (o con la realidad); o, de forma más precisa, una teoría es verdadera si, tan solo si, corresponde a los hechos" (1985:51). En La lógica de la investigación científica, Popper -citando al filósofo Reichenbach- escribe: "hemos descrito el principio de inducción como el medio por el que la ciencia decide sobre la verdad. Para ser más exactos, deberíamos decir que sirve para decidir sobre la probabilidad: pues no le es dado a la ciencia llegar a la verdad ni a la falsedad..., mas los enunciados científicos pueden alcanzar únicamente grados continuos de probabilidad, cuyos límites superior e inferior, inalcanzables, son la verdad y la falsedad" (1985:29) y destaca que la verdad "es la correspondencia con los hechos". Inspirado en el concepto aristotélico de verdad, y en declaraciones posteriores del matemático y filósofo Alfred Tarski, argumenta que la característica fundamental de su tesis es que recupera la noción clásica de verdad, entendida como correspondencia de los hechos. Su propuesta de falsacionismo significa que la ciencia no puede poseer la verdad absoluta y sus enunciados no son verificables, ni probables, sino falsables.

En un nivel ontológico Popper se basa en los hechos. Según sus palabras: "Una proposición es verdadera si, y solo si, corresponde a los hechos" (1985:53). El conocimiento que poseemos de la realidad subjetiva es limitado. Aprendemos a ver la "realidad", seleccionando los mensajes que llegan a nuestro conocimiento subjetivo de esta realidad, la que consiste en descifrar a través de la "eliminación de experiencias" y de errores. Debido a la dificultad de buscar la verdad, Popper, observa la necesidad de que "por lo menos en algunos casos" (1985:53) pueda ser lo 
más próximo posible de la verdad, a partir de la actualización de una determinada teoría o de una teoría "mejor". Por fin, Popper advierte que, en todo caso, somos procuradores de buscar la verdad, "pero no somos sus poseedores", ya que la verdad nunca se expone con exclusividad. Lo máximo que podemos conseguir es -según Popper- llegar lo "más próximo posible" a la verdad. Podemos concordar en parte con estas explicaciones basadas en la visión de los hechos, en las cuales aparece la necesidad de contar con un instrumento para aproximarnos a la realidad: las hipótesis y "aserciones" -entendidas como argumentos que se dan por conceptos- y la necesidad de refutarlas o de aceptarlas.

Con los argumentos dados por Popper, es posible establecer tres aspectos de carácter metodológico-epistemológico que conforman la búsqueda de la verdad en la investigación científica social: la forma de producción del conocimiento y la adquisición del saber, el método científico y la validez de los resultados. Para reflexionar sobre estos aspectos consideramos otras dos variables que en cierta forma están contenidas transversalmente en las descritas por Popper: la primera de carácter teórico y epistemológico, como es la perspectiva del género y de las mujeres. En cuanto a la segunda, comprende las condiciones sociales en que se produce el conocimiento. Lo que entendemos por conocimiento popular, a primera vista, no se diferencia del conocimiento científico, ni por la necesidad, ni por naturaleza del objeto. Lo que básicamente diferencia ambos tipos de conocimiento es el método y los instrumentos para conocer los fenómenos y la verdad.

\subsection{La concepción positivista y materialista: la búsqueda de la realidad}

La perspectiva materialista en el nivel epistemológico, al descubrir los hechos, se apoya en el positivismo y en la clásica definición de Durkheim sobre la estrategia de investigación, al definir los fenómenos sociales como "cosas" y la observación de los "hechos", entendidos estos como una "manera de actuar" y con una existencia propia "independientemente de sus manifestaciones individuales" (Durkheim 1960:93). Para Durkheim, la tarea de las ciencias sociales es mostrar que las uniformidades y las regularidades de la conducta humana no son accidentales. Para analizar estas uniformidades es necesario poseer un "método apropiado a la naturaleza de las cosas estudiadas y a los requisitos de la ciencia" (Durkheim 1960:9). Esta perspectiva científica apoyada en la similitud que establece entre las ciencias sociales y naturales, se basa en la aceptación de leyes naturales y en la necesidad de corroborarlas mediante un proceso empírico. Los científicos sociales analizan los sistemas sociales, apoyados en la observación de la estructura de las diferentes partes que los componen y que interpretan a partir del análisis de las partes, las cuales se encuentran en conexión y pueden ser delimitadas en su entorno. Las ciencias sociales extrajeron normalmente sus teorías a través de un proceso de observación de la realidad, elaboración de hipótesis, contrastación, demostración o no de las mismas hipótesis, y construcción de modelos o teorías generales. El paradigma positivista o naturalista ofreció el marco y los límites de análisis de la realidad como una "cosa" externa al individuo.

El materialismo histórico dialéctico se inspiró en la idea dialéctica original de Hegel: lo que es racional se hace real, y lo que es real se hace racional. La idea indica que cualquier fenómeno no es racional solo por el hecho de existir. La teoría marxista centró su foco de estudio y analizó de forma prioritaria el sistema económico y las estructuras sociales y de poder que causan las desigualdades sociales y, en consecuencia, la exclusión social y la pobreza, especialmente la que se refiere a la exclusión económica. La teoría marxista clásica partió de la perspectiva estructuralista, o sea, es el sistema, en el caso actual, el neoliberal, que "determina" esta situación de desigualdad. El ser humano se encuentra dentro de una situación objetiva, en este caso la estructura social -o en palabras de Max Weber "una jaula de hierro"- en que su acción es determinada por esta estructura, consecuentemente limitada. Esta forma de pensamiento puede ser resumida como: "La historia nos muestra el hombre como una totalidad de relaciones con el mundo y con otros hombres, de acuerdo con su situación objetiva en la estructura social. En esta relación -o en estas 
relaciones- se construyen los medios y los fines de sus acciones" (Faleiros 1982:60). A partir de esta descripción, el ser humano es interpretado a partir de una situación objetiva dada por leyes que rigen su acción. La categoría central de totalidad comprende las relaciones entre los hombres insertos dentro de una situación objetiva de la estructura social, la cual es determinante. En este sentido, la estructura social tiene un papel predominante en la conciencia de los individuos, y en consecuencia en la acción y conducta de ellos, los cuales están en gran medida determinados por las estructuras.

Para el marxismo estructuralista, la observación de la realidad debe centrarse en las estructuras o sistemas que se forman a partir de la interacción de las relaciones sociales. La perspectiva de la corriente principal de la teoría marxista adopta el razonamiento dialéctico. Los elementos decisivos en estas lecturas, a veces mecanicistas de Marx, fueron la lectura e interpretación, en ocasiones interesadas, del sentido original que dio Marx a los conceptos de infra-estructura y superestructura. En este sentido, las traducciones e interpretaciones realizadas por Harnecker relacionan la infra-estructura solamente a las condiciones económicas de la sociedad: “El nivel económico -la forma en que los hombres producen los bienes materiales y las relaciones que se establecen entre ellos en el proceso de producción" (1986:28). La idea original de Marx difiere de estas traducciones que reducen su obra a un carácter básicamente mecanicista, y por lo tanto no dialéctico, puesto que él afirmaba que el pensamiento humano se fundamenta en la actividad humana, en especial en el trabajo. Sin embargo, el entendimiento que Marx da al trabajo es en un sentido amplio, o sea, él contempla las relaciones sociales derivadas del trabajo, con lo cual incluye la socialización o la interacción social. Transformar o realizar un cambio en la infra-estructura tendría -según la misma autora- consecuencias directas para la superestructura "el nivel jurídico-político-ideológico". Esta lectura ha tenido consecuencias en la práctica partidista de los partidos comunistas latinoamericanos. Para Marx, el pensamiento económico se fundamenta en la actividad humana, siendo el trabajo y las relaciones sociales lo que constituyen la infra-estructura, o, en sus palabras, "la actividad humana". La superestructura, por lo tanto, es el mundo producido por tal actividad. Para Marx, la actividad humana implica una compleja serie de interacciones entre los seres humanos.

El carácter mecanicista dominó al explicarse estos fenómenos sociales como consecuencia de la estructura de poder burgués y de la desigualdad social. Las investigaciones sobre la sociedad de carácter no económico fueron insuficientemente analizadas por los científicos sociales. Las categorías desarrolladas algunas décadas antes por Gramsci y su concepto de "societá civile" y "arena política" contribuyeron con elementos analíticos para la conquista de la arena política de estudios de la sociedad, en un momento en que la situación de democratización política y conquista de espacios democráticos de los países de América Latina lo requería.

La teoría marxista ha dado mucha importancia al estudio de la actividad humana y al trabajo desde la perspectiva de la subjetividad. Solamente durante la década de los años 30, los científicos y filósofos que formaron parte de la Escuela de Frankfurt contribuyeron con elementos necesarios para analizar el ser humano a partir de otras perspectivas no economicistas, al incorporar los elementos psicológicos y culturales en los mecanismos de dominación capitalista. El diálogo sistemático con la fenomenología se realizará cuatro décadas más tarde.

La idea de los paradigmas dominantes de concebir a la clase trabajadora como el motor de cambio social se cuestionó con la aparición de otros actores sociales a partir de mediados de la década de los años 1960. Los movimientos feminista, ecologista, de homosexuales, negros, minorías étnicas y estudiantes, formados básicamente por la clase media, no surgieron a partir del análisis de las contradicciones económicas y sociales, sino a partir de la comprensión subjetiva de las contradicciones sociales de sus participantes. Este fenómeno expone y abre un debate sobre la acción y la estructura, o sea, sobre el actor principal impulsor del cambio social en las sociedades avanzadas. 
Para las corrientes del materialismo histórico posterior a Marx, la dialéctica materialista constituye la fuente del conocimiento, la que se basa principalmente en los escritos de Marx, Engels y Lenin. Para Lenin, en el libro Materialismo y empiriocriticismo, el conocimiento surge de un proceso de constante contradicción histórica: "El conocimiento es la aproximación eterna, infinita, de pensamiento al objeto. El reflejo de la naturaleza en el pensamiento del hombre debe ser entendido en eterno proceso de movimiento, de surgimiento de las contradicciones y de su solución". Su obtención no corresponde a una evolución lineal o acumulativa. Más tarde en En torno a la cuestión dialéctica Lenin escribe: "El conocimiento del hombre no es una línea recta, sino una curva que se aproxima infinitamente a una serie de círculos, una espiral". Lenin también entró en el debate ontológico, al sentar las bases de una teoría del conocimiento materialista dialéctico. La componen dos enunciados principales. El primero referente a la existencia de cosas independientemente de nuestra consciencia y de nuestro sentimiento, y externa a los sujetos. El segundo, relativo a la no existencia de ninguna diferencia entre el fenómeno y la cosa en sí. Existe diferencia simplemente entre lo que es conocido y lo que todavía está por conocer. Los hombres se encuentran inmersos en esta contradicción, la de investigar el mundo y sus relaciones y la propia naturaleza. Pero el hombre tendrá sus límites en esta búsqueda llena de contradicciones, debido a la existencia de leyes universales y de su propia naturaleza. Entretanto, esta contradicción no está presente solo entre el mundo y el hombre, al existir siempre en un nivel histórico, aunque Engels amplía el determinismo histórico en Dialéctica de la Naturaleza al afirmar que esta contradicción se manifiesta subjetivamente por la textura física y espiritual de su autor.

¿Es posible conocer la verdad? Es un punto central de la teoría materialista dialéctica. El materialismo dialéctico parte de leyes ya existentes, tanto a nivel subjetivo como a nivel social, formulándose de tal forma que las siguientes preguntas son inevitables: ¿qué leyes tiene el hombre, nuestra consciencia y el mundo que nos rodea? Estas cuestiones aparecen de forma transversal en la teoría del conocimiento. El concepto de verdad se concretiza en la verdad objetiva, la cual no depende del hombre, denominada verdad subjetiva. La verdad subjetiva existe, una vez que su contenido depende del hombre. Por otro lado, la verdad objetiva no es algo dado, estático, mas es entendida como un proceso que incluye fases cualitativas. Para el materialismo histórico, la verdad se encuentra entre la verdad absoluta y la verdad relativa.

\subsection{La interpretación comprensiva de la realidad}

Al final de la década del 70 e influenciada por los movimientos sociales, surgió en las universidades californianas una nueva corriente epistemológica denominada etnometodología. La emergencia de esta corriente se explica a partir del auge propio del movimiento estudiantil en muchas ciudades como Paris, Berlín, São Paulo, Barcelona, México y el lento declinar del movimiento obrero tradicional. Si los estudiantes representaban la élite económica y la burguesía, cómo explicar que fueran éstos los que intentaban promover cambios sociales y revolucionarios en las universidades, ¿qué sucedió con el proletariado y los asalariados, como agentes históricos tradicionales y con los partidos comunistas?

En un nivel de análisis sociológico teórico, y en ese contexto social de protestas y de emergencia de los nuevos movimientos sociales, la etnometodología surgió como respuesta a la teoría dominante del funcionalismo, que sostiene que las motivaciones de los actores están integradas a modelos normativos que regulan las conductas y las apreciaciones recíprocas. La emergencia de los Nuevos Movimientos Sociales como el movimiento feminista, los ecologistas o los pacifistas, como los tres grandes ejes en los que se constituyen otros movimientos sociales, confirmó la tendencia de entender la política en primera persona, o sea, la subjetividad a través de la experiencia.

Hemos expuesto de forma sucinta cómo en las últimas décadas la subjetividad se ha manifestado en el plano político con la representación de "política en primera persona" característica de los movimientos sociales. El plano político 
aparece como prioritario para la observación de los que denominados "realidad", con el objetivo de cambiarla o modificarla. Para este artículo de carácter introductorio y para la operacionalidad de las observaciones sistémicas, aparecen dos cuestiones centrales: ¿qué noción filosófica sobre el término verdad nos puede orientar en la observación de la complejidad? y ¿cómo se establece una relación epistemológica entre la observación y la realidad?

Dado que entre los teólogos, filósofos y lógicos a lo largo de los siglos la "verdad" ha sido considerada un tema concerniente al alma y a la psicología racional, tratándose de un tema claramente metafísico y por lo tanto no central para las cuestiones que planteamos, ofrecemos de forma sucinta una aproximación a la terminología. Para el positivismo lógico (Otto Neuraht, Rudorf Carnap), la "verdad" es algo que está ahí afuera, es "dada”, y nosotros solo tenemos que descubrirla y copiarla. Para el humanismo pragmático de William James, la verdad depende en general del sujeto que pone a prueba una idea, para poder decir que el enunciado, o la idea expresada por este enunciado, tenga la propiedad de ser verdadera.

La filosofía trascendental de Robert Sokolowski, inspirado en la fenomenología de Martin Heidegger, constata que en la vida subjetiva y racional de los individuos, tienen lugar dos tipos de verdad: la verdad "exactitud" y la verdad "descubierta" (2012:169). La verdad de "exactitud" se inicia con una proposición (o enunciado) que deberá ser verificada. Caso la proposición o hipótesis sea confirmada, podemos afirmar que la proposición es verdadera ya que expresa la forma de como son los hechos. Se trata según Sokolowski de una afirmación exacta. El sentido de la falsedad que es correlato a la verdad es obvio, ya que será la falsedad de las proposiciones o hipótesis que se han formulado de forma opuesta de como son los hechos o estos se manifiestan. Sin embargo, constata la existencia de una forma más elemental de procurar la verdad, y que puede hasta separar la conformación de la proposición (hipótesis). Se trata de la verdad "descubierta", o sea, de la exposición de un estado de cosas. La falsedad de este tipo de verdad ocurre en el momento en el que las apariencias engañan, o sea, cuando las cosas parecen ser lo que en realidad no son. La verdad de "exactitud" depende de la verdad "descubierta", siendo esta última la que sirve para confirmar o negar una proposición. En los dos tipos de verdad expuestas, el predicado "verdadero" se ha aplicado tanto a una proposición como a un estado de cosas.

Con el término "realidad" nos referimos a describir cómo la entendemos para la adquisición de conocimiento en el contexto de la idea que proponemos de autopoiesis reflexiva y desde la perspectiva del observador. La pregunta central fue formulada por José Ferrater Mora: ¿existe una realidad cuya esencia es hacer posible y real la existencia de lo que individualmente existe, o es simplemente un concepto abstracto? Nos remitiremos a la sociología fenomenológica y la descripción dada por Alfred Schütz.

Las raíces epistemológicas de la teoría de Alfred Schütz claramente están en la fenomenología de Edmund Husserl, el cual considera que todas las ciencias, se trate de las ciencias naturales o humanas, son una "totalidad" de las actividades humanas. Para Husserl, en toda ciencia, la base de sentido es el "mundo de la vida" (Lebenswelt) precientífico, que es el mundo mío y de todos nosotros. Schütz también está influenciado por la concepción pragmatista de la "realidad" del matemático y filósofo Alfred Whitehead, y del humanismo de William James, ya que ambos reconocen la existencia de una realidad social. Schütz subjetiviza la operación de existencia de esta realidad, al otorgarle un origen claramente subjetivo al fenómeno "real", en el momento de poseer una cierta relación con el sujeto: "la realidad significa simplemente una relación con nuestra vida emocional y activa" (Schütz 1971:197). Otorga a los sentidos y a las experiencias captadas por el sujeto la fuente principal para la percepción de la realidad: "es el sentido de nuestras experiencias, y no la estructura ontológica de los objetos, lo que constituye la realidad" (Ibid.: 303). Sin embargo, constata diferentes tipos de realidad, al no considerarla única, monolítica y homogénea, sino estructurada en órdenes diversos en los cuales tiene su propio estilo especial y separado. Con ello también 
reconoce la existencia de una realidad externa al individuo. De forma similar a la descripción de Edmund Husserl, Schütz considera que los hechos y fenómenos externos se construyen en nuestra conciencia como "objetos ideales", al ser internalizados en nuestra mente y reconstruidos subjetivamente por nosotros. Su significado está determinado por la relación que el actor establece con los objetos o fenómenos observados. Como resultado, su composición es intersubjetiva. Lo que observamos, sea cosa u objeto, no es solo producto de nuestros sentidos, sino que además posee un componente hipotético.

En la internalización de la realidad, las raíces epistemológicas provienen de los conceptos elaborados por Schütz de "mundo social" y "mundo de vida". En el primero, las personas usan tipificaciones, o sea, acciones que vienen determinadas por un tipo constituido por experiencias anteriores. El ser humano tipifica su propia situación dentro del mundo social, con sus semejantes y con el mundo de los objetos culturales. Realiza una analogía de un libro de recetas culinarias, en el que las actividades prácticas están establecidas mediante recetas. En situaciones problemáticas las personas crean nuevas recetas válidas para manejarse. En el segundo concepto, "mundo de vida" (que Schütz también denominó a lo largo de su obra como "mundo del trabajo cotidiano" o "realidad eminente del sentido común"), se trata del mundo en el que la intersubjetividad y las recetas de los sujetos se aplican. Cada sujeto posee un mundo de vida propio, puesto que no existe un mundo único, aunque pueden existir elementos comunes entre los diferentes sujetos. El "mundo de vida" ya existe antes de nuestro nacimiento, puesto que son las instituciones las principales transmisoras de este mundo. Con ello indica la existencia de una dialéctica de influencia mutua entre los actores y las estructuras.

Un paso decisivo en la sociología comprensiva es dado en la obra de Max Weber. El fundamento hay que buscarlo en las leyes de la razón, compuesta por la ley de la homogeneidad y la ley de la afinidad. Este última se refiere que para entender los fenómenos, hay que colocarlos en relación de afinidad con otros fenómenos, como es el caso de la economía con las estructuras jurídicas o religiosas. Sin embargo, la sociología fenomenológica de Schütz se centra en el análisis de la intersubjetividad, aunque en este autor adquiere una lectura sociológica, que paulatinamente Peter Berger y Thomas Luckmann contribuyen a popularizar. La perspectiva interpretativa ve la necesidad de comprender las situaciones particulares por medio de los pormenores de la vida práctica, además de considerar qué significados adquieren para las personas los acontecimientos. La aceptación teórica del conocimiento sobre el carácter de la realidad social adquiere una dimensión específica con el constructivismo. Schütz ya constató el hecho de que el significado y la interpretación de los hechos es la base del constructivismo: "Todos los hechos son el resultado de un contexto universal escogido, a través de los hechos seleccionados por los procesos de nuestra conciencia. Con ello se trata siempre de hechos interpretados: o son separados de su contexto, abstracciones abstractas, o son vistos solo en su particular contexto. En ambos casos los hechos apoyan la interpretación en un horizonte interno y externo" (Schütz 1971:5).

De la descripción y significado de la realidad y de la forma en que se accede al mundo y a la realidad a través de la conciencia, se desprende el fundamento del constructivismo. ¿Es todo hecho construido por la conciencia? A pesar de la radicalidad de la tesis de Schütz, no todos los científicos que siguen en mayor o menor medida el constructivismo comparten esta opinión. En este sentido algunos filósofos y científicos consideran que la conciencia, manifestada en la epoché de Husserl, aborda la percepción como la "relación directa entre la conciencia y el mundo" (Osorio 1998:15), mientras que Glasersfeld (1996:30) argumenta que "ningún constructivismo defiende la opinión de que todo es construido". Incluso el denominado constructivismo radical no niega la existencia de una realidad externa. La percepción del mundo no es un proceso de recepción pasiva, sino que se trata de un proceso de construcción activa. El aceptar esta premisa abre la cuestión sobre si una representación de la realidad puede ser comprobada en su veracidad original. Para la teoría constructivista del conocimiento y la investigación empírica, es el saber y las 
correspondientes construcciones que se derivan, un camino relevante para acceder a los hechos con lo que se ocupan.

Aunque la obra de Schütz La Fenomenología del Mundo Social se publicó en 1932, se tendrá que esperar hasta 1967 para ser traducida al inglés y hasta 1978 para que se publique una coetánea de su obra en portugués y en castellano. La conciencia es para Schütz el punto de partida: ¿cómo conocemos otras mentes?, ¿cómo se produce la reciprocidad de perspectivas? Su preocupación central es la conciencia, en especial las estructuras universales de la conciencia. La conciencia y los procesos de interpretación de la conducta y de los hechos. Para Schütz, los hechos se apoyan en la interpretación subjetiva que realiza el hombre a través de la conciencia.

Su obra se vuelve mundialmente reconocida, gracias en parte a su difusión para un público más amplio por Peter Berger y Thomas Luckmann con el libro La Construcción Social de la Realidad. Aunque no resulta fácil describir las ideas básicas de la teoría, es posible enumerar una serie de principios básicos (siguiendo a Ritzer 1993:237): A diferencia de los animales inferiores, los seres humanos están dotados de capacidad de pensamiento. La capacidad de pensamiento está modelada por la interacción social. En la interacción social las personas aprenden los significados y los símbolos que les permiten ejercer su capacidad de pensamiento distintivamente humana.

Los elementos constitutivos de la fenomenología y del interaccionismo simbólico sustentan que las personas pueden procurar otras vías a las de supeditarse en las relaciones de autoridad, puesto que el individuo en su vida cotidiana o en su mundo de vida construye sus propias relaciones sociales. Es consciente, entretanto, del peso constrictivo de las estructuras sobre los individuos en tres aspectos. En primer lugar, la constricción material, que deriva del carácter del mundo físico y del propio cuerpo. Segundo, la constricción ligada a las sanciones, o sea, las respuestas punitivas y sancionadoras que realizan unos agentes sobre otros. Tercero, la constricción estructural, derivada del contexto de la acción y de las propiedades estructurales frente a los actores. Se parte de la premisa de que la estructura no tiene que identificarse con la coacción, cuyo argumento parte de tres ideas centrales:

1) La estructura es entendida como un elemento que constriñe a los individuos, pero también como un elemento que facilita la acción.

2) No debe establecerse una identificación que lleve a una distinción entre la acción y la estructura, un vínculo entre diferentes niveles macro sociológicos y micro sociológicos.

3) La acción no puede vincularse automáticamente con las intenciones, entendiendo la acción como la capacidad que poseen los individuos para realizar cosas.

Desde sus inicios, el interaccionismo simbólico y la etnometodología fueron sistemáticamente cuestionadas por su extremo subjetivismo, ya que no analizaban las estructuras sociales causadoras de las desigualdades. Entretanto, la teoría y la práctica demostraban un déficit analítico e instrumental para analizar cada situación particular del actor o del sujeto. En las sociedades avanzadas surgieron fenómenos vinculados a prejuicios de carácter xenofóbico o racista, sobre las opciones sexuales personales, la violencia doméstica, las diversas opciones filosóficas y religiosas, los procesos de aculturación, el análisis feminista del patriarcado, entre otros. Estos fenómenos fueron interpretados a partir de la necesidad de construir una sociedad socialista en la que no existirían estas marcas discriminatorias de orígenes burguesa y capitalista. La realidad de la vida cotidiana, de la propia evolución de las sociedades y de las experiencias del socialismo real, muestra, entretanto, la insuficiencia de este posicionamiento. 
El interaccionismo simbólico sitúa como tema central la influencia de los significados y de los símbolos sobre la acción y la interacción humana. Los interaccionistas, como Herbert Blume, se oponen a las teorías sociologistas -en especial al funcionalismo estructural- pero también a las diferentes corrientes estructuralistas que consideran que la conducta individual está determinada por macro fuerzas exteriores o factores socio-estructurales tales como el sistema social, la estructura social, las costumbres, el papel social y los valores. Se enfatiza la subjetivación, entendida como un proceso de símbolos y significados como elemento central de formación del ser humano, como contrapuesta a la observación instrumental de carácter positivista. "El mundo de la vida cotidiana" y la experiencia subjetiva de la vida cotidiana, en palabras de Berger y Luckmann, son la realidad por excelencia, puesto que es en la intersubjetividad de la vida cotidiana donde los seres humanos proporcionan las subjetivaciones necesarias para su convivencia.

Una posición muy diferente asume Hans-Georg Gadamer en Verdad y Método, donde advierte a no caer en un error: "Será la ciencia, como ella propia reivindica para sí, la última instancia y la única portadora de la verdad" (Gadamer 2004:58). Es evidente que debemos al desarrollo de la ciencia el hecho de habernos podido libertar de prejuicios, tabúes, a medida que se ha desarrollado en sus procedimientos y en la comprensión de "lo real".

\subsection{Teoría de sistemas autorreferenciales y teoría holística}

La obra de Luhmann se vincula a una serie de estructuras conceptuales en las cuales la circularidad es asumida, si no siempre como una virtud argumentativa, casi siempre como un modelo heurístico de carácter general y fecundo. ¿Cómo se puede observar el desarrollo y la interrelación de la técnica desde la perspectiva del observador, cuando él mismo está inmerso en la observación del desarrollo? Para intentar ofrecer una respuesta, parece necesario abrir un debate teórico sobre la observación. Parte de la idea (en cierta forma constructivista-fenomenológica) según la cual "toda observación es relativa al punto de vista del observador", aunque Luhmann amplía la idea con la posibilidad de observación del propio observador. Para Luhmann la observación sobrepasa las diferencias clásicas de vivencia, acción o psíquicas y asume "todo tipo de operación que consuma una diferencia para indicar así una parte (y no otra)" (1984:97).

Esta tesis no lleva a formular dos cuestiones: ¿cómo podemos delimitar y establecer el sistema? y ¿cómo podemos establecer las distinciones? y aunque Luhmann aporta una serie de explicaciones teóricas a estas cuestiones, como es la operación de definir el sistema del entorno, o sea, una distinción en la que se divide el mundo, debemos referirnos a la teoría de las distinciones, elaborada por Spencer Brown. Para mostrar su teoría Brown se fundamenta sus postulados en la lógica matemática y algebra lineal. En Laws of Form de 1969, escribe que toda forma surge del establecimiento de una distinción, o sea, de un gesto o acción que establece un dentro, un afuera y unos márgenes. En este sentido, la forma en tanto que distinción contiene a su vez y distintamente lo que abarca como lo que no abarca. De ello se puede derivar que la distinción, como continencia necesaria, solo puede ser la "contingencia perfecta", o sea, contiene a su vez la forma distinta, aquello que abarca y que se diferencia de aquello que no abarca. Es posible deducir que la distinción aparece como instituyente con la fundación previa de la forma y en este encuentro es esencial para su comprensión. Para Brown: "La forma requiere de la difícilmente previsible intervención disposicional de un agente humano o no humano en un contexto marcado por la pluralidad y complejidad de fuerzas". La consecuencia de ello es la posibilidad de desprender la existencia de un mundo sin acoplamientos y sin la existencia de repertorios y disposiciones. Sin embargo, alejándonos momentáneamente de la abstracción teórica, no puede darse ninguna forma sin la intervención de un agente que no sólo realiza la acción de aplacar la distinción, sino de construir y modificar una relación más compleja.

Para Luhmann no se trata como "debe ser" el fenómeno observado, en el sentido otorgado por Habermas, sino como "es" en este momento observado, por lo que el papel del observador es esencial para evitar que afecte al sistema 
observado. El análisis de la complejidad social solo puede realizarse bajo la abstracción en el nivel de la complejidad teórica, cuyo centro es el sistema, interpretado como un conjunto organizado de relaciones técnico-estructurales, dotadas de propiedades, capaces de supervivencia y autogobierno -autorreferenciales (autopoiéticas).

Para Luhmann, la observación sociológica empírica tradicional habitualmente cae en una paradoja que sugiere una serie de situaciones. Estas aparecen en el momento de observar un hecho social en su funcionamiento normal, puesto que en el momento de la operación de observar, se interfiere en el hecho social y éste deja de representar el funcionamiento. Por lo tanto, el observador necesita previamente definirse respecto al observado, constituyendo en este acto una continuidad de procesos comunicativos. Estos se manifiestan en la delimitación del objeto de investigación y en los métodos y técnicas utilizados para ser aplicados.

Para evitar esta paradoja e interferencias en las observaciones, Luhmann establece una diferenciación entre la observación elemental y la observación de segundo orden. Con las observaciones de tipo elemental se trata básicamente de la utilización de códigos binarios. En ella, todo puede ser observado, siempre y cuando utilice la distinción, o incluso observe las propias distinciones, siendo la aplicación de códigos binarios la lógica metodológica que propone Luhmann. En la observación de "segundo orden", u observación de observaciones, se trata de una observación que se realiza sobre el observador. Dada la multiplicidad y pluralidad de sistemas autopoiéticos, operando cada uno de estos en virtud de observaciones, se hace necesario recurrir a la observación de observaciones, u observación de "segundo orden". Siguiendo el principio sistémico de Luhmann, "no puede operar ya bajo premisas ontológicas, puesto que estas presuponen que lo que existe es o que es" (Luhmann 1988:33). Esta tesis tiene consecuencia metodológica. En primer lugar (que él mismo diferencia), la observación elemental permite operar bajo premisas ontológicas. En segundo lugar, este tipo de observación necesita de una “lógica multivalente” (Ibid. 33), para la observación de los sistemas. Con ello, Luhmann abre la posibilidad de utilizar diversas lógicas metodológicas, sean cuantitativas o cualitativas, como es el caso de la fenomenología.

Posteriores aportaciones a la teoría sistémica, desde la perspectiva de la politextualidad y de la "godelización", abren una perspectiva y una nueva mirada sobre las tecnologías sociales. Específicamente en las corrientes: análisis sociotécnico y redes técnico-económicas (Callon); el ensamble socio-técnico, políticas de la investigación científica y tecnológica (Bijker); la adecuación socio-técnica (Thomas) y la economía del cambio tecnológico y trayectorias tecnológicas (Freeman, Dosi). Basadas en datos empíricos de la realidad y con ello cuantificables, hay un predominio de técnicas estadísticas y matemáticas. Sin embargo, estas áreas de conocimiento, aunque direccionadas al pensamiento interdisciplinar, manifiestan su incapacidad para incluir y comprender la acción subjetiva, de tal forma que aparece necesario observarlas desde la perspectiva de una "epistemología reflexiva” (Zolo).

En una perspectiva de crítica a la regularidad y a las leyes que determinan las estructuras sociales, Edgar Morin, basado en Marcel Mauss y sus ideas de "hombre total" y "hecho social total", analiza los presupuestos y las bases en las que se apoyan las ciencias sociales y sociedades posindustriales. Para Morín la sociología dominante: "reduce la sociedad a la noción de sociedad industrial (o pos-industrial), circunscribe el singular concepto en monografías descriptivas y elimina pura y simplemente el conocimiento, considerado como un accidente. Para superar esta situación de poca creatividad y conocimiento alineado y procurar la verdadera realidad social, "es necesario tomar distancia del fenómeno para concebir la verdadera realidad social" (1998:166).

De nuevo, encontramos la necesidad de analizar la sociedad y los fenómenos sociales a partir de un conocimiento que no se apoye en un determinismo y un reduccionismo del conocimiento, en el sentido de que un conocimiento del todo ejerza de punto de partida para un conocimiento de las partes que lo componen. Morin (2000), por el contrario, sugiere la "necesidad de recomponer el todo", o sea, el cuestionar la racionalidad abstracta y 
unidimensional hegemónica. Iniciado por Max Weber y posteriormente por la escuela fenomenológica, aparece la necesidad de procurar un nuevo pensamiento. El filósofo y sociólogo francés Edgar Morin (1998) en la década de los años setenta introdujo la necesidad de desarrollar esta nueva forma de pensar a partir de un pensamiento que postula: (a) que el captar el conocimiento de las partes, depende del conocimiento del todo (holismo) y que el conocimiento del todo depende del conocimiento de las partes, (b) que reconozca y trate los fenómenos multidimensionales en vez de aislar de manera mutiladora cada una de sus dimensiones, (c) que reconozca y trate las realidades que son a la vez solidarias y conflictivas (como la democracia misma, que se nutre de antagonismos al mismo tiempo que los regula) y (d) que respecte lo diverso, al mismo tiempo que reconoce lo único.

Afirma que un pensamiento que aísla y separa hay que sustituirlo por un pensamiento que distinga y una. Se hace necesario desarrollar una epistemología de la complejidad, que se basa en la teoría del caos y la teoría de sistemas. Según Morin (2000), en las sociedades racionales actuales, el elemento del caos y la incertidumbre aparecen constantemente. La distancia entre el orden y el desorden se ha reducido a un mínimo. En adelante conceptos como los de estructuras emergentes o disipativas, historia del sistema, cambios catastróficos o caos, no evocan la periferia de la comprensión de la organización social, sino que quedan implicados en la comprensión de la lógica de su funcionamiento general. La existencia de estructuras disipativas y relaciones miméticas o de contagio entre los comportamientos de los sistemas sociales proporciona una nueva forma de transdisciplinariedad entre la psicología de las masas, las teorías acerca del individuo y de los sistemas sociales y las modernas investigaciones en física y biología. La operacionalización de esta idea se fundamenta en tres principios. El principio dialógico: superación de los antagonismos en una construcción superior. El principio de recurrencia: los efectos circulares y en forma de bucle que afectan a todo fenómeno humano. El principio hologramático: el todo está en la parte, del mismo modo que esta se encuentra en el todo.

El conocimiento acerca de los fenómenos complejos implica inseguridades y desconocimiento parcial, principalmente acerca de los niveles diferenciados de su organización o del sistema de interacción entre ellos o entre niveles epistemológicos diversos, basados en el Principio de Pascal. En los últimos años se sigue discutiendo sobre la forma de operacionalizar el paradigma de la complejidad. Las propuestas aportan reflexiones sobre una nueva forma de realizar la lectura de los datos estadísticos, a partir de propuestas como es el análisis residual (Lovatto, Lehnen, Andretta, Carvalho, Hauschild). Desde una perspectiva en la que domina la estadística, se proponen una serie de niveles posibles para la investigación: i) Análisis exploratorio. Supone hacer una inmersión en los datos y que expresen su riqueza con el objetivo que hagan evidentes determinadas relaciones y patrones; ii) Meta análisis. Conduce hacia una teoría probabilística global. Se pueden utilizar alternativas explicativas y comprensivas; iii) Simulaciones informáticas. Aplicación de simulaciones informáticas en el análisis social, consensuan la abertura de nuevas alternativas a las formas tradicionales de investigar.

Las implicaciones metodológicas de estos pensamientos resultan importantes. Para Luhmann su estrategia de investigación no es "ni inductiva ni deductiva". La estrategia de obtención de datos está configurada por el tipo de observación, entre la observación de primer grado y la observación de segundo grado. Para los presupuestos holísticos de Morín aparece también la problemática en torno a la metodología y las técnicas más adecuadas para la procura de la verdad, de tal forma que algunos autores afirman que el "análisis crítico de una metodología holística utópica sugiere una revisión del término cualitativo" (Martínez Miguelez 2000:40). Ambas cosmovisiones están estrechamente vinculadas con el debate cualitativo-cualitativo, considerado como "falso" o "superado" (Brüsemeister, Flick, Kelle, Ersberger). 
La procura de la verdad es el objetivo de toda observación. Específicamente para la observación de carácter sistémico, la propuesta de Sokolowski referente a la reflexión del término verdad de "exactitud" no resulta el más adecuado, dado que se trata de una lógica fundamentada en la linealidad de la observación, o sea, en la formulación de hipótesis causales. Por otro lado, el sentido semántico atribuido a la verdad "descubierta", o sea, la exposición de un estado de cosas, nos permite su utilización operativa en la lógica de sistemas autopoiéticos, puesto que esta terminología permite el desvelar constante de las apariencias de lo que se denomina "realidad exterior".

En forma de síntesis, en las ciencias sociales habitualmente se ha utilizado en la observación de la realidad la lógica de pensamiento lineal. Sin embargo, como hemos expuesto, la lógica "circular" característica de la teoría de sistemas, o la lógica "policontextual" propuesta por Luhmann (1984:11), ofrece posibilidades todavía no exploradas para las observaciones. Esta lógica es una opción clara de colocar entre paréntesis lo que entendemos por realidad material -o la realidad "allí fuera"-, construida por el consenso hegemónico. Puede que esta posición nos aproxime a posiciones cercanas a un solipsismo, pero abre interesantes interrogantes que colocan la autorreflexidad (en un sentido sistémico, o aunque a partir de posiciones diferentes, en un sentido fenomenológico), al observador como centro de la observación.

\subsection{La teoría autopoiética: ¿realidad objetiva o construida?}

La teoría autopoiética se trata de un método de observación social y significa la capacidad de producirse a sí mismo. El término original ofrece, según el neurobiólogo Francisco Valera, una salida para la superación de los obstáculos de las ciencias cognitivas al ofrecer una respuesta a la intencionalidad. Valera define un sistema autopoiético como: "aquel que continuamente produce los componentes que lo especifican al tiempo que lo realizan (el sistema) como unidad concreta en el espacio y el tiempo" (Valera 1992:2). Valera matiza que la autopoiesis de los sistemas vivos posee una identidad, puesto que el concepto: "pretende capturar los mecanismos o procesos que generan la identidad de lo vivo (...) frente a lo no-vivo" (Valera 1992:3). Esta aclaración permite abrir la puerta epistemológica sobre la identidad de los sujetos, aunque sigue siendo claramente anclada en la sociobiología.

El título en forma de pregunta del libro de Humberto Maturana La realidad: ¿objetiva o construida? indica con claridad reflexiones teóricas sobre la posibilidad de poner en cuestión lo que habitualmente denominamos como realidad. La pregunta central que formulamos es: ¿Cómo se configura el conocimiento? La respuesta es muy amplia y permite dar diferentes respuestas y explicaciones, puesto que toda teoría o modelo teórico depende de la respuesta a la cuestión planteada. Para Maturana: "las explicaciones son proposiciones presentadas como reformulaciones de experiencias que son aceptadas como tales por un oyente" (1995:73). De ello, se deriva el reconocimiento de que hay tantos tipos de explicaciones, como también hay tantos diferentes criterios que pueden ser aceptados como diferentes tipos de experiencias. Para Maturana, la lógica que precede a la respuesta hay que buscarla en la forma histórica de cómo se ha construido la teoría del conocimiento. La idea cuestiona los fundamentos de la comprensión de la realidad desde el siglo XVI. Desde ese siglo, se ha fundamentado en torno a dos ejes: el racionalismo y el empirismo.

La perspectiva sociobiologista de Maturana sostiene que la "realidad es irreconocible", debido a las denominadas "trampas del conocimiento". La primera "trampa" es la creencia de que el mundo material de los objetos puede dar instrucciones al conocimiento. Sin embargo, la procura del conocimiento indica dos posibles "trampas". La primera referida a la forma de ejecutar la diferenciación sistémica y su determinación por la "clausura operacional" sistémica. La segunda "trampa" se deriva de la primera, al atribuir a la clausura una soledad cognoscitiva cuyas consecuencias son la exclusión de la operación de organizar el mundo. El resultado de estas ideas es que el conocimiento se configura por medio de un "acoplamiento estructural". La decisión presupone que el conocimiento, como una 
operación autopoiética, opera como un sistema desde su interior mediante sus propias estructuras. Con esta tesis, se excluye que datos procedentes o existentes en el entorno, puedan especificar lo que sucede en el sistema.

\section{Implicaciones metodológicas en la adquisición del conocimiento}

Como fue expuesto, la realidad social puede ser observada a partir de formas diferentes, como el comportamiento individual y social. En las ciencias sociales, se desarrollaron dos formas que se pueden sintetizar de la siguiente manera: una primera sustenta que las ciencias sociales son semejantes a las ciencias naturales, y por consiguiente se preocupan de descubrir las leyes universales que determinan el comportamiento individual y social, como acontece en el mundo animal. Las ciencias sociales, por lo tanto, se limitan a describir una situación. La segunda, el denominado idiográfico, en que me apoyo en este escrito, sustenta que la ciencia social se preocupa básicamente en explicar cómo las personas interpretan estos fenómenos. De estas dos visiones se basan en diferentes formas de entender el comportamiento humano, y de observar la realidad social, y en consecuencia se manifiestan en la forma de investigar, sea en estudios sociológicos, pedagógicos educativos o politológicos, por dar algunos ejemplos.

El supuesto ontológico de relación con otros seres humanos y con el entorno, se apoya en que el ser humano puede ser objeto y sujeto de la investigación y del estudio, de ello se derivan dos imágenes de ser humano. Por un lado, se entiende la actuación del ser humano como producto de su entorno, condicionado por las circunstancias y las estructuras dominantes y en que la persona responde mecánicamente a su entorno. En un nivel filosófico, es interpretado como un determinismo. Por otro lado, el ser humano es el iniciador de sus propias acciones, crea su entorno, el libre arbitrio es el centro de su actuación, atribuyéndose un "papel creativo". Los científicos, y las teorías elaboradas, están arraigados en mayor o menor medida en cada uno de estos supuestos, los cuales tienen en su conjunto implicaciones para el observador y para la forma de abordar los métodos y las técnicas de investigación. El observador adopta una postura "objetiva" o "subjetiva” en la observación, ya que los distintos supuestos de carácter ontológico, epistemológico, o de relación con el entorno, exigirán también métodos de investigación diferentes.

A menudo las observaciones son motivadas por consideraciones ideológicas, sin embargo, solo serán válidas, como Bunge recuerda, si se "ajustan” al método científico. Mediante la aplicación del método científico se podrá obtener el conocimiento científico. Jürgen Habermas propone tres formas de investigación que abarcan todos los ámbitos de la actividad humana: 1) El “interés técnico". La orientación general de las ciencias de la naturaleza se fundamenta en un interés de raíces antropológicas, que tiene como objetivo la predicción y el control de los fenómenos que tienen lugar en el mundo natural. 2) La "orientación general” con la finalidad del autoconocimiento y la comprensión. 3) El "interés por la emancipación", para logar el dominio natural por medio del control. En Conocimiento e Interés adelanta en su tesis principal que se trata de una "crítica radical al conocimiento y que solo es posible con una teoría social" (Habermas 1982:9), refiriéndose a la estrecha interconexión existente entre el método de procura del conocimiento y la cosmovisión filosófica y teórica. Formula dos cuestiones centrales: ¿qué son y cómo se aplican los intereses cognitivos? Esta última para descubrir el conocimiento humano: ¿qué importancia tiene la dominación y la comunicación? A partir del interés de cada categoría como determinante de la forma con que puede "observarse la realidad" y de las cuestiones planteadas y los tipos de interés que expone, enumera tres tipos de investigación: ciencias empírico-analíticas, ciencias histórico-hermenéuticas, ciencias de orientación crítica. Para Habermas, las teorías son sistemas de enunciados que pueden ser correctos o incorrectos y por lo tanto permiten ser corroborados o falsados: "incluye no solamente las teorías científicas formuladas con cierto rigor, sino también al conjunto de afirmaciones y suposiciones, explicitas o implícitas, sobre la base de las cuales un investigador establece sus hipótesis o realiza sus inferencias" (Garcia 2000:44). 
Otros autores al tratar las formas en que el hombre puede adquirir conocimiento o procurar la verdad, proponen una clasificación básicamente en tres amplias categorías: la experiencia, el razonamiento y la investigación (Cohen y Manion, Mouly). Estas categorías no son entre sí independientes ni excluyentes, de forma que el paso de una a otra no está delimitado, apareciendo como un continuum. En la primera categoría, la experiencia, existen por lo menos cuatro formas que el ser humano utiliza para adquirir el conocimiento: la primera es el método de la tenacidad, la segunda el método de la autoridad, la tercera el método a priori y el cuarto el método científico (Arnau, Kerlinger). Los tres primeros métodos son considerados como no científicos por la falta de sistematización y control, mientras que el cuarto método es el propiamente científico. Para que un método sea científico ha de comprender una serie de etapas básicas o principales. Bertrant Russell asevera que para llegar a establecer una ley científica son necesarias tres etapas: la primera consiste en observar los hechos significativos; la segunda, en sentar hipótesis que, si son verdaderas, expliquen aquello hechos; la tercera, en deducir de estas hipótesis consecuencias que puedan ser puestas a prueba para la observación. Las etapas están inseridas en un contexto científico, ya que las hipótesis no están aisladas sino que existen dentro del cuerpo general del conocimiento científico. Significa que el proceso de su formulación debe ser justificado y explicado paso a paso, para de esta forma construir y formular hipótesis que sean contrastables. Para Russell, en toda investigación sobre la naturaleza de la verdad se tropieza con dos cuestiones: i) ¿en qué sentido, si es que hay alguno, la verdad depende del espíritu?, ii) ¿hay muchas verdades diferentes, o existe solamente La Verdad? (Russell 1972:188, itálicas mías). Sugiere que la segunda cuestión parece más fundamental, al estar relacionada con el monismo ontológico, o sea la reducción de los hechos y las cosas a un principio unitario regido por el ser, entendido este como la síntesis de la sustancia del alma y del cuerpo. La implicación metodológica de este presupuesto, significa cuestionar la división entre ciencias de la naturaleza y ciencias del espíritu, ya que ambas son interpretadas como una, o sea, orientadas hacia un monismo epistemológico.

En el momento de planear el método para una observación existe no obstante también la posibilidad de que se confunda método con teoría. Filósofos de la ciencia (Bunge, Popper) y científicos (Latorre, Arnal) definieron la teoría científica y lo que diferencia la teoría de las simples especulaciones, pues una especulación sería como imaginar algo sin el fundamento necesario.

Por otro lado, la teoría de sistemas autorreferenciales de Niklas Luhmann se fundamenta en la teoría de sistemas, aunque adquiere una característica propia al ser aplicada a la sociedad. El análisis funcional-estructuralista de Luhmann, no consiste en establecer las conexiones entre los datos establecidos o el conocimiento confiable a través del cual, como consecuencia, se obtiene conocimiento adicional; se preocupa finalmente de los problemas y su solución. Por lo tanto, el método "no es ni deductivo ni inductivo sino más bien heurístico en un sentido particular" (Luhmann 1996:7). Deja claramente entrever que no se trata de la observación tradicional con el objetivo de determinar la causa-efecto de un fenómeno, como sucede en la teoría clásica de sistemas, sino que con los métodos heurísticos se trata de un conjunto de reglas metodológicas, generalmente basadas en reglas empíricas de estadística avanzada y algoritmos (Bueno) que sugieren un papel específico del observador. Las observaciones pueden ser definidas como estudios de caso, y el método que sugiere es “básicamente matemático" (Luhmann 1996:79), aunque en la idea dicotómica de la observación elemental predominan códigos binarios, mientras que en la observación de segundo grado posibilita la metodología cualitativa basada en códigos emergentes. De las premisas metodológicas expuestas, se deduce que la finalidad de la estrategia metodológica es el "acceso a una realidad situada en el exterior" (Luhmann 1996:159). Los problemas se plantean en términos de la "conservación de la estabilidad de los sistemas de acción; o más abstractamente de la identidad; de la definición del mundo real” (Luhmann 1996:7). 


\section{¿Es posible establecer conclusiones?: la búsqueda de la verdad como proposición kantiana}

La búsqueda de la realidad se realiza mediante métodos basados en el positivismo, dominante en las ciencias sociales y humanas, mientras que la procura de la verdad se realiza mediante juicios apriorísticos. La primera tiene como objetivo observar el mundo real. Se trata de un mundo construido a partir del consenso colectivo, basado en construcciones sociales. La segunda, la procura de la verdad, se trata de una construcción subjetiva, que pone en cuestión el mundo material de las apariencias, habitualmente ignorada, puesto que se construye a partir del lenguaje y la interacción. Sin embargo, consideramos que entre el construccionismo radical de Peter Berger al afirmar "las cosas no son lo que parecen" (Berger 1985:40) o "solamente un bárbaro intelectual es capaz de afirmar que la realidad es únicamente lo que podemos ver mediante métodos científicos" (Berger 1985:41) y la relación constatada por Marx en Manuscritos Económico y Filosóficos (Primer Manuscrito, Trabajo alienado, XXIV, 1844) entre "la construcción práctica de un mundo objetivo" y el trabajo ejercido en este mundo donde el hombre se comporta como un "ente-especie", aparece una dificultad epistemológica para resolver en el nivel ontológico las cuestiones planteadas. Los intentos de un marxismo de orientación fenomenológica apenas han podido responder satisfactoriamente las cuestiones ontológicas y epistemológicas que se plantean.

Para alcanzar la procura de la verdad, o las verdades, es evidente que cada ser humano posee una vivencia subjetiva que determina la "visión del mundo" o Weltanschauung. A partir de la forma como entendemos la realidad y el mundo que rodea a los seres humanos, han emergido diversas formas de comprenderlo. De la hipótesis se derivan una serie de cuestiones epistemológicas: ¿es la comprensión neutra?, ¿es el conocimiento neutral? Las respuestas son innumerables y no conclusivas, pues dependerán de la concepción del mundo de cada sujeto. Giulio Giardi, desde su perspectiva del conocimiento como instrumento de transformación social, denuncia la falsa neutralidad de la ciencia y del conocimiento, puesto que todo sujeto en el momento de la observación forma parte de una serie de condicionantes y estructuras internalizadas: "No hay interés teórico que esté desvinculado de intereses prácticos. El desinterés en el ocultamiento de intereses que no quieren confesarse (...) el conocimiento no puede ser juez sin ser parte" (1977:101). De esta aseveración, se deriva que son cuestionables las concepciones e instrumentos aceptados comúnmente, y los parámetros dominantes de la ciencia. Aceptar esta idea tiene consecuencias en las formas de conocer el mundo, al tratarse, según Moreno, "producidas por y para dominarlo (las formas de saber vinculadas al ejercicio del poder), se han legitimado históricamente como expresión de lo real... a base de definir como apariencia, opinión, engaño y hasta superstición e ignorancia, cuantas formas de conocimiento no se ajustan a sus criterios valorativos y sus reglas" (1991:62).

La perspectiva expuesta induce a una relación específica de objeto y sujeto, en la que se cuestiona la forma holística, la separación estricta entre sujeto y objeto de investigación. La teoría crítica defiende que el objetivo final del conocimiento es la emancipación, entendida como el asumir el control de la propia vida en el nivel subjetivo y colectivo, o sea la autorreflexión y reflexión sobre el conocimiento en el sentido dado por Habermas. La reflexión es una característica de la reflexividad, al hacer parte el investigador del escenario en que desarrolla la investigación en esta dirección teórica.

En la pregunta ontológica ¿hay posibilidad de encontrar alguna salida?, Platón expone la posibilidad de que la realidad puede ser expresada en dos dimensiones. La primera, el mundo de los sentidos, es el mundo de la percepción. La segunda, el mundo de las ideas, representa la forma ideal del mundo de los sentidos. En el mundo de las ideas se trata de un mundo de formas perfectas y que puede ser percibido como un mundo ideal, en el que el ser humano es único. Veinte siglos más tarde, Richard Rorty propone el concepto medio de la existencia en el que armonizan lo material y lo abstracto. 
A nuestro entender, no es si la realidad es construida o "externa" material, sino en qué nivel nos estamos refiriendo: en un nivel de análisis instrumental sobre la necesidad de políticas públicas, en el que los indicados cuantitativos son necesarios, o en un nivel subjetivo -o de observador- de búsqueda de las inseguridades construidas del hombre del siglo XXI, con él mismo y con su entorno. Si nos referimos a este último, deberemos procurar la verdad o posibles verdades.

\section{Bibliografía}

Archer, M. 1988. Culture and agency: the place of culture in social theory. Cambridge: Cambridge University Press.

Berger, P. 1985. La reinterpretación de la sociología. Madrid: Espasa-Calpe

Durkheim, E. 1960. As regras do método sociológico. São Paulo: Cia. Editora Nacional.

Faleiros, V. 1982. Metodologia e ideologia do trabalho social. São Paulo: Cortez editora.

Gadamer, H-G. 2004. Verdad y método II. Fundamentos de una hermenéutica filosófica. Salamanca: Sígueme.

Giardi, G. 1977. Por una pedagogía revolucionaria. Barcelona: Laia.

García, R. 2000. El conocimiento en construcción. De las formulaciones de Jean Piaget a la teoría de los sistemas complejos. Barcelona: Gedisa.

Glasersfeld, E. von. 1996. Construtivismo radical, una forma de cohecer e aprender. Lisboa: Instituto Piaget.

Habermas, J. 1982. Conocimiento e interés. Madrid: Taurus.

Harnecker, M. 1986. Qué es la sociedad. Santo Domingo: Editora Taller.

Luhmann, N. 1998. Complejidad y modernidad: de la unidad a la diferencia. Madrid: Trotta.

Luhmann, N. 1996. Confianza. Barcelona: Anthropos.

Luhmann, N. 1984. Soziale systeme. Grundisse einer Allgemeinen Theorie. Frankfurt a.M.: Suhrkamp.

Martínez Miguelez, M. 2000. El paradigma emergente: hacia una nueva teoría de la racionalidad científica. México: Trillas.

Maturana, H. 1995. La realidad: cobjetiva o construida? Fundamento biológicos de la realidad. Barcelona: Anthropos.

Maturana, H. y Valera, F. 1985. El árbol del conocimiento: Las bases biológicas de entendimiento humano. Santiago: Editorial Universitaria.

Morin, E. 1998. Introducción al pensamiento complejo. Barcelona: Seix Barral.

Morin, E. 2000. La mente bien ordenada. Barcelona: Seix Barral.

Moreno, A. 1991. Pensar la historia a ras de piel. Barcelona: Tempestad. 
Osorio, F. El método fenomenológico: aplicación de la epoché al sentido absoluto de la conciencia. Cinta de moebio 3: 50-63.

Popper, K. 1985. La lógica de la investigación científica. Madrid: Tecnos.

Ritzer, G. 1993. Teoría sociológica contemporánea. Madrid: McGraw-Hill.

Russell, B. 1972. Ensayos filosóficos. Madrid: Alianza Editorial.

Schütz, A. 1971. El problema de la realidad social. Buenos Aires: Amorrortu editores.

Sokolowski, R. 2012. Introducción a la fenomenología. Morelia, Michoacán: Editorial Jitanjáfora.

Valera, F. 1992. Autopoiesis and a biology of intentionality. En: McMullin, B. and Murphy, N. (eds.) Autopoiesis \& perception. Dublin: Dublin City University, pp. 1-14.

Recibido el 14 Mar 2013

Aceptado el 25 Jul 2013 